\title{
Performance Evaluation of Fixed Sample Entropy for Lung Sound Intensity Estimation
}

\author{
Manuel Lozano-García, Jasna Nuhić, John Moxham, Gerrard F. Rafferty, Caroline J. Jolley, and \\ Raimon Jané, Senior Member, IEEE
}

\begin{abstract}
Lung sound (LS) signals are often contaminated by impulsive artifacts that complicate the estimation of lung sound intensity (LSI) using conventional amplitude estimators. Fixed sample entropy (fSampEn) has proven to be robust to cardiac artifacts in myographic respiratory signals. Similarly, fSampEn is expected to be robust to artifacts in LS signals, thus providing accurate LSI estimates. However, the choice of fSampEn parameters depends on the application and fSampEn has not previously been applied to $\mathrm{LS}$ signals. This study aimed to perform an evaluation of the performance of the most relevant fSampEn parameters on LS signals, and to propose optimal fSampEn parameters for LSI estimation. Different combinations of fSampEn parameters were analyzed in LS signals recorded in a heterogeneous population of healthy subjects and chronic obstructive pulmonary disease patients during loaded breathing. The performance of fSampEn was assessed by means of its cross-covariance with flow signals, and optimal fSampEn parameters for LSI estimation were proposed.
\end{abstract}

Clinical Relevance - LSI is considered to be an indirect measurement of the airflow entering the lungs, so that LSI can be used to detect airway obstruction and assess lung disorders that affect ventilation. The clinical potential of LSI analysis depends, however, on the ability to obtain accurate estimates of LSI. The fSampEn parameters proposed in this study allow fSampEn to be optimally applied to LS signals, thus contributing to improving LSI estimation in health and disease.

\section{INTRODUCTION}

Respiratory sounds (RS) are produced during breathing by airflow patterns within the airways [1]. Normal RS are random in nature and their characteristics depend on the recording site. RS recorded at the chest surface, that is lung sounds (LS), have a frequency band from around $70 \mathrm{~Hz}$ to around $1000 \mathrm{~Hz}$, with a sharp decrease in power above $250 \mathrm{~Hz}$ [2]. Lung sound intensity (LSI) is affected by respiratory diseases that alter airflow patterns and regional ventilation. Indeed, decreased LSI is associated with airflow limitation, which in turn can be due to airway conditions, such as chronic obstructive

This work was supported in part by the Generalitat de Catalunya (CERCA Program and GRC 2017 SGR 01770), in part by the Gobierno de España (RTI2018-098472-B-I00 MCIU/AEI/FEDER, UE), and in part by the Biomedical Research Networking Centre in Bioengineering, Biomaterials and Nanomedicine (CIBER-BBN, Instituto de Salud Carlos III/FEDER). M. Lozano-García was the recipient of a European Respiratory Society Fellowship (ERS LTRF 2015-5185).

M. Lozano-García, J. Nuhić, and R. Jané are with the Institute for Bioengineering of Catalonia (IBEC), The Barcelona Institute of Science and Technology (BIST), UPC Campus Diagonal-Besòs, Av. d'Eduard Maristany, 10-14, building C, 5th floor, 08930, Barcelona, Spain (e-mails: mlozano, pulmonary disease (COPD) [3]. LSI, quantified as the amplitude of LS signals, has been proposed as an indirect measurement of the airflow entering the lungs [4], and can therefore be used to detect unilateral or bilateral lung disorders. LS signals, however, are often highly contaminated by impulsive artifacts, such as clicks, bursts, scratches, or cardiac artifacts. These events make it difficult to obtain accurate estimates of LSI using conventional amplitude estimators, such as RMS, thus limiting the clinical potential of LS analysis.

Fixed sample entropy (fSampEn) has been proposed as a means to estimate respiratory muscle activity from electromyographic and mechanomyographic signals, with less interference from cardiac artefacts [5]-[7]. fSampEn is based on sample entropy, which measures regularity of signals [8], so that more regular and predictable signals have lower values of sample entropy. In sample entropy, two input parameters must be selected: $m$ (embedding dimension) and $r$ (tolerance parameter). The latter is generally expressed as a function of the standard deviation of the signal under analysis, which in this study is an LS signal (LS_SD). In fSampEn, sample entropy is not calculated at once for a whole signal, but within a moving window, using a fixed $r$ for all windows. In this way, fSampEn is sensitive to changes in signal amplitude [5]. In myographic respiratory signals, fSampEn has proven to be very robust to cardiac artifacts, which are more predictable and regular than electromyographic and mechanomyographic signals [5], [6].

As in myographic respiratory signals, fSampEn is expected to be robust to artifacts in LS signals, thus providing more accurate LSI estimates as compared to RMS. However, fSampEn has not previously been applied to LS signals. Despite the potential for using fSampEn to analyze LS signals, fSampEn can vary significantly with the choice of $m, r$ and window length, which in turn depends on the application. While $m$ is typically fixed at 1 or $2, r$ and window length are more critical for the magnitude and shape of fSampEn [9],

jnuhic, rjane \{@ibecbarcelona.eu\}). M. Lozano-García and R. Jané are also with CIBER-BBN and Universitat Politècnica de Catalunya (UPC)Barcelona Tech.

J. Moxham, G. F. Rafferty, and C. J. Jolley are with the Centre for Human \& Applied Physiological Sciences, School of Basic \& Medical Biosciences, Faculty of Life Sciences \& Medicine, King's College London, King's Health Partners, London, United Kingdom (e-mails: john.moxham, gerrard.rafferty, caroline.jolley \{@kcl.ac.uk\}). G. F. Rafferty and C. J. Jolley are also with King's College Hospital NHS Foundation Trust, King's Health Partners, London, United Kingdom. 
[10]. Therefore, there is need for research on the performance of fSampEn in LS signals.

The aim of this study was to conduct, for the first time, an evaluation of the performance of fSampEn in LS signals, which led us to propose optimal fSampEn parameters for LSI estimation.

\section{Methodology}

\section{A. Study Population}

LS and respiratory flow were recorded in 35 subjects, including 21 healthy subjects (11 male, age 42 (33-64) years, body mass index $23.7(21.8-26.0) \mathrm{kg} / \mathrm{m}^{2}, \mathrm{FEV}_{1} / \mathrm{FVC} 80.3$ (74.4-83.1) \%) and 14 COPD patients (9 male, age 68 (65-72) years, body mass index $25.5(19.4-28.0) \mathrm{kg} / \mathrm{m}^{2}, \mathrm{FEV}_{1} / \mathrm{FVC}$ $38.2(30.0-46.6) \%)$. This study was conducted at the King's College Hospital, London, United Kingdom. The study was approved by the NHS Health Research Authority (NRES Committee London-Dulwich 05/Q0703) and the experiments conformed to the standards of the Declaration of Helsinki. All subjects were fully informed of any risk associated with the study and provided their written consent before participation.

\section{B. Data Acquisition}

LS were recorded using four piezoelectric contact microphones (TSD108, BIOPAC Systems Inc.) placed bilaterally on the skin over the posterior base (left (LL) and right (LR)) and upper lobe (left (UL) and right (UR)) of the lungs. Airflow was recorded using a pneumotachograph (4830, Hans Rudolph Inc.) connected to a differential pressure transducer (DP45, Validyne Engineering). Mouth pressure $\left(\mathrm{P}_{\mathrm{mo}}\right)$ was measured from a side port on the pneumotachograph using a second differential pressure transducer (MP45, Validyne Engineering). LS signals were amplified by 100 before acquisition (CED 1902, Cambridge Electronic Design Limited). All signals were acquired using a 16-bit analogueto-digital converter (PowerLab 16/35, ADInstruments Ltd.) at $100 \mathrm{~Hz}$ (flow and $\mathrm{P}_{\mathrm{mo}}$ ) and $10000 \mathrm{~Hz}$ (LS). After acquisition, LS signals were resampled at $4000 \mathrm{~Hz}$, band-pass filtered between $70 \mathrm{~Hz}$ and $1950 \mathrm{~Hz}$, and filtered with a comb notching filter to remove the power line interference at $50 \mathrm{~Hz}$ and all its harmonics.

A maximal static inspiratory pressure (PImax) maneuver [11] was performed initially, sitting upright with a noseclip in place, and repeated several times to ensure maximal volitional effort. All subjects performed then an inspiratory threshold

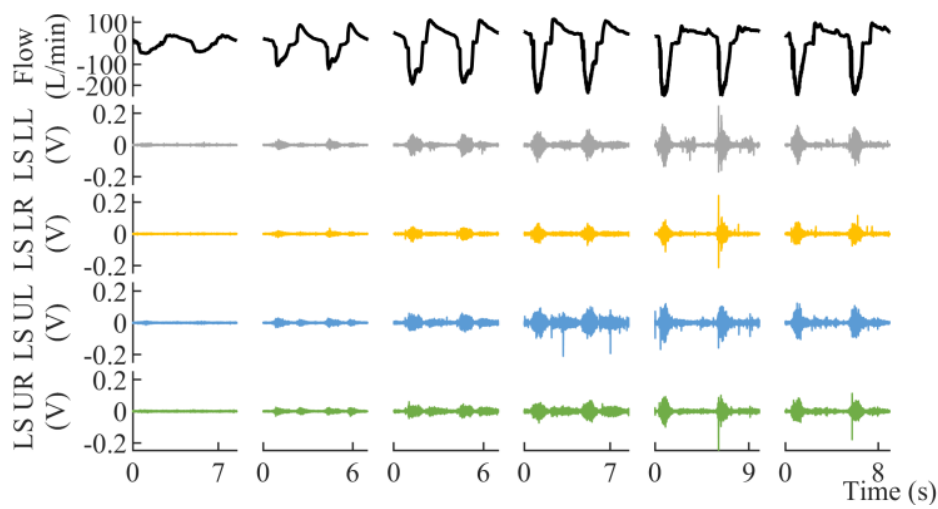

loading protocol at five inspiratory threshold loads set at $12 \%$ $(L 1), 24 \%(L 2), 36 \%(L 3), 48 \%(L 4)$, and $60 \%(L 5)$ of the subject's PImax. Inspiratory threshold loads were generated using an electronic inspiratory muscle trainer (POWERbreathe K5; POWERbreathe International Ltd.) attached to the distal end of the pneumotachograph. Subjects were seated and breathed through the pneumotachograph via a mouthpiece with a noseclip in place. Baseline measurements were recorded during a minimum of 2 minutes of quiet resting breathing $(L 0)$, following which the POWERbreathe was attached to the pneumotachograph and the loads were imposed. Each load consisted of 30 breaths followed by a resting period to allow all respiratory measures to return to baseline.

\section{Data Analysis}

All signals were segmented into inspiratory and expiratory segments by means of a zero-crossing detector on the $\mathrm{P}_{\mathrm{mo}}$ signal. Since LS are much louder during inspiration than during expiration, only inspiratory segments were analyzed in this study.

Cycle selection: 60 inspiratory segments per subject were selected as follows:

- The following parameters were calculated for all inspiratory segments: inspiratory time $(T i)$, inspiratory volume $(V i)$, peak flow $(F p)$, and maximum absolute value of the corresponding four LS signals (S1-S4). Then, the median parameters of all inspiratory segments within each load $L i$ were calculated.

- The absolute differences $\left(d_{T i}, d_{V i}, d_{F p}, d_{S I}-d_{S 4}\right)$ between all seven parameters and its corresponding median values were calculated for all inspiratory segments. For each inspiratory segment, all the absolute differences were normalized to their maximum values within the corresponding load.

- The absolute differences of each inspiratory segment were averaged as in (1).

$$
d_{G l o b}=\left(4 d_{T i}+4 d_{V i}+4 d_{F p}+d_{S 1}+d_{S 2}+d_{S 3}+d_{S 4}\right) / 16
$$

- The 10 inspiratory segments with the lowest $d_{\text {Glob }}$ were selected for each load $L i$.

Global LS SD calculation: In order to use fSampEn as an LSI estimator, fSampEn time-series of different LS signals should be comparable, and therefore must be calculated using

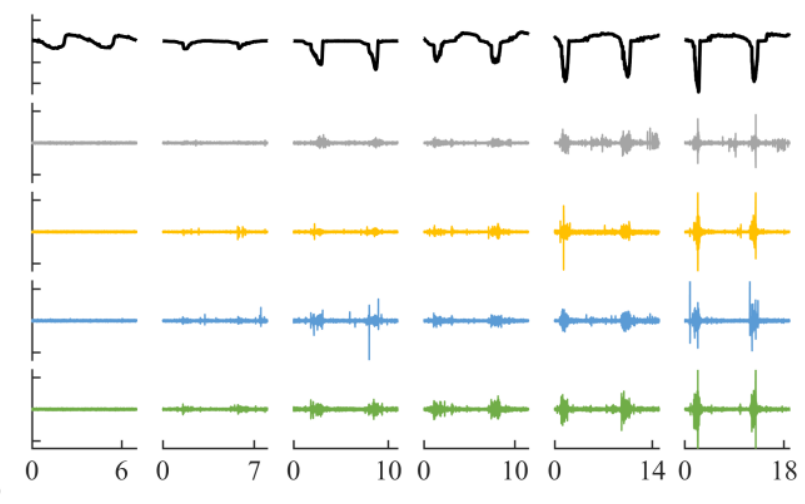

Figure 1. Flow and LS signals recorded during the inspiratory threshold loading protocol in a healthy subject (left) and a COPD patient (right). Two respiratory cycles are shown for quiet breathing and threshold loading. Negative flow values correspond to inspiratory phases. 
the same fSampEn parameters. Since $r$ is dependent on the LS_SD of the LS signal analyzed, a unique global LS_SD of the four LS signals acquired at the different loads and in all subjects is required to calculate a unique global $r$ value. Firstly, LS_SD values were calculated individually for the 60 inspiratory segments and the four LS signals of each subject. Then, LS_SD values of all subjects were averaged to obtain a unique global LS_SD.

fSampEn calculation and evaluation: fSampEn time-series of a subject's four LS signals acquired during resting breathing and the inspiratory threshold loading protocol were calculated using $m$ equal to 2, window length ranging from $0.05 \mathrm{~s}$ to $0.5 \mathrm{~s}$ in increments of $0.05 \mathrm{~s}$, and tolerance set as $r$ times the unique global LS_SD, with $r$ ranging from 0.05 to 0.6 in increments of 0.05 . A $90 \%$ overlap between adjacent windows was used. As a result, a total of $2880 \mathrm{fSampEn}$ timeseries were calculated for each subject. To make this feasible, fSampEn time-series were calculated on a subset of 5 healthy subjects and 5 COPD patients randomly chosen from the study population. Similarity between fSampEn time-series and flow signals was assessed as the maximum crosscovariance, $c_{\max }$, of the inspiratory segments of fSampEn time-series and flow signals.

\section{RESULTS}

Fig. 1 shows representative recordings from a healthy subject and a COPD patient. As observed, the four LS signals of each patient are very similar, and inspiratory LS are higher than expiratory LS.

\section{A. LS_SD Analysis}

LS_SD values are shown in Fig. 2, for each threshold load and the four LS signals of both healthy subjects and COPD patients. Inter-load variability of LS_SD values was very clear, since airflow increased with increasing load, and therefore so did LS. However, LS_SD increased less in patients than in healthy subjects. In any case, LS_SD values of all four LS signals were very similar within each load.

A unique global LS_SD value of 0.0085 was calculated by averaging all LS_SD values shown in Fig. 2. This global LS_SD value was used as a fixed reference for the $r$ parameter in the calculation of fSampEn time-series.

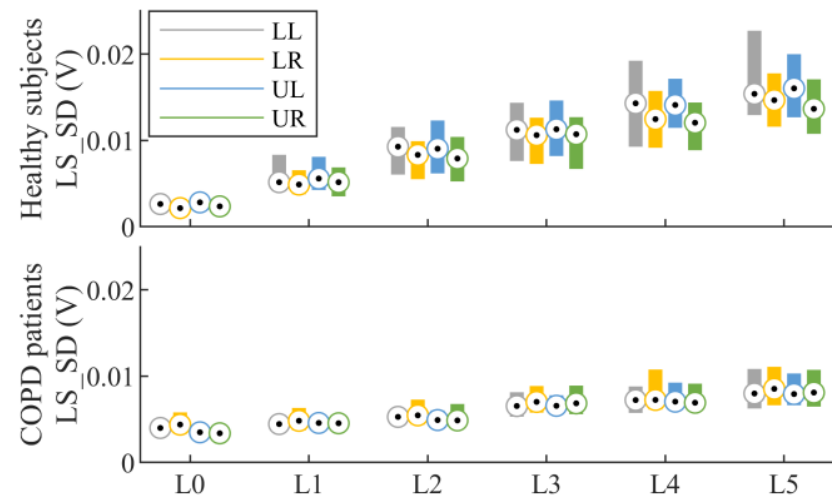

Figure 2. Median and interquartile range of the LS_SD of the four LS signals recorded in healthy subjects (top) and COPD patients (bottom) during quiet breathing and threshold loading.
TABLE I. $\quad C_{M A X}$ Values Averaged Over All LS Signals

\begin{tabular}{|l|c|c|c|c|c|c|}
\hline \multicolumn{1}{|c|}{ Subject } & L0 & L1 & L2 & L3 & L4 & L5 \\
\hline Healthy 1 & 0.42 & 0.83 & 0.70 & 0.93 & 0.89 & 0.93 \\
\hline Healthy 2 & 0.56 & 0.63 & 0.73 & 0.83 & 0.87 & 0.89 \\
\hline Healthy 3 & 0.35 & 0.51 & 0.87 & 0.86 & 0.85 & 0.85 \\
\hline Healthy 4 & 0.67 & 0.67 & 0.75 & 0.83 & 0.93 & 0.91 \\
\hline Healthy 5 & 0.46 & 0.76 & 0.79 & 0.90 & 0.90 & 0.92 \\
\hline COPD 1 & 0.29 & 0.37 & 0.53 & 0.59 & 0.71 & 0.68 \\
\hline COPD 2 & 0.21 & 0.48 & 0.69 & 0.88 & 0.93 & 0.91 \\
\hline COPD 3 & 0.36 & 0.83 & 0.84 & 0.94 & 0.85 & 0.83 \\
\hline COPD 4 & 0.45 & 0.32 & 0.55 & 0.89 & 0.62 & 0.70 \\
\hline COPD 5 & 0.76 & 0.37 & 0.48 & 0.51 & 0.78 & 0.76 \\
\hline All & 0.44 & 0.56 & 0.68 & 0.81 & 0.82 & 0.83 \\
\hline
\end{tabular}

\section{B. Performance of fSampEn}

Table I shows the $c_{\max }$ values averaged over all LS signals for each subject in each threshold load. In general, $c_{\max }$ increased with increasing threshold load.

Fig. 3 shows the $c_{\max }$ values averaged over all threshold loads, all LS signals and both healthy subjects and COPD patients. The highest $c_{\max }$ value (0.68) was obtained for a window length of $0.35 \mathrm{~s}$ and $r$ equal to 0.2 .

\section{DISCUSSION AND CONCLUSIONS}

In this study, we have conducted an evaluation of the performance of fSampEn, for the first time, for LSI estimation, which led us to propose the following optimal fSampEn parameters adapted to LS signals: $0.35 \mathrm{~s}$ moving window with $0.3 \mathrm{~s}$ overlap, $m$ equal to 2 , and $r$ equal to $0.2 \times 0.0085$.

Estimating LSI using conventional amplitude estimators is difficult due to the high presence of impulsive noise in LS signals. Previous studies have proposed the use of Shannon entropy in a moving window as a means to analyze tracheal RS signals, for breath-phase onset estimation [12], [13], apnea detection [14], or flow estimation [15]. Shannon entropy, however, uses the probabilities of occurrence of the data analyzed, which must be calculated in advance. Contrarily, fSampEn can be directly applied to the data without the need for any prior calculations, while also being very stable to large outliers or artifacts. Although fSampEn has been previously used in acoustic breathing signals for apnea detection [16], its use as an estimator of LSI is unexplored.

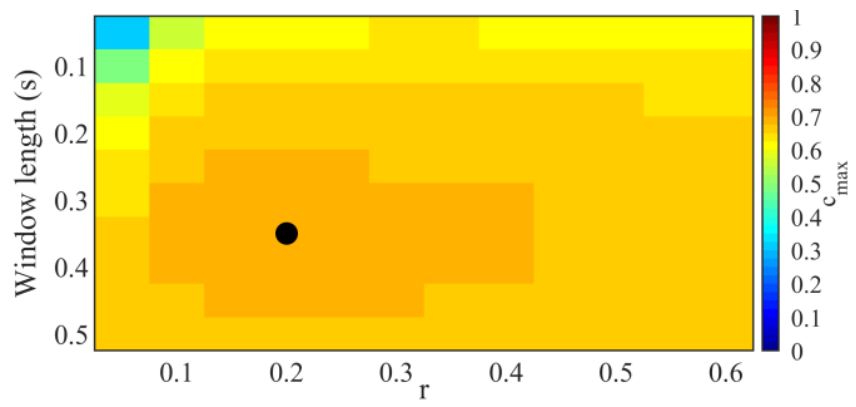

Figure 3. Similarity $\left(c_{\max }\right)$ between inspiratory segments of flow signals and fSampEn time-series of the four LS signals recorded in healthy subjects and COPD patients during quiet breathing and threshold loading. The black dot corresponds to the highest $c_{\max }$. 

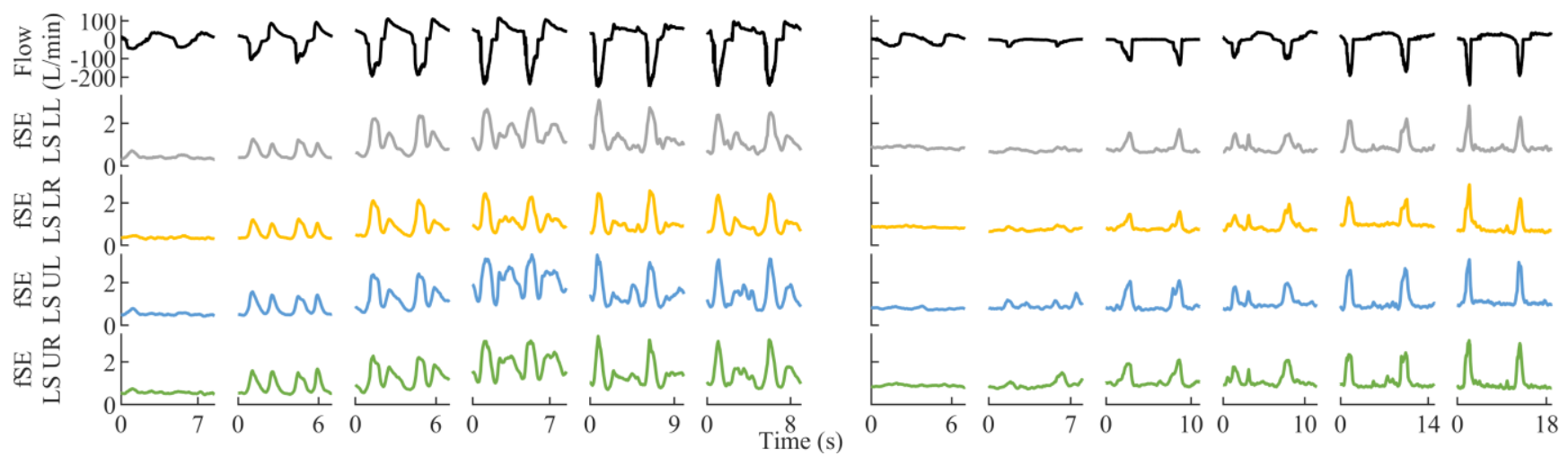

Figure 4. fSampEn time-series (fSE) of the signals shown in Fig. 1, recorded during the inspiratory threshold loading protocol in a healthy subject (left) and a COPD patient (right). fSampEn time-series were calculated using the optimal fSampEn parameters proposed in this study.

In this study, we have investigated the performance of fSampEn for LSI estimation in a wide variety of LS signals. Firstly, LS signals were recorded at several points over the chest surface. This multichannel analysis of LS is important, since LS characteristics may vary with the recording position. Secondly, the study population included both healthy subjects and COPD patients, and therefore provided LS signals related to both normal and impaired ventilation. Furthermore, the inspiratory threshold loading protocol provided the potential to acquire LS signals under a wide range of flow levels. Due to the close relationship between LSI and respiratory flow, the performance of fSampEn as an estimator of LSI was assessed by means of the cross-covariance of fSampEn time-series and flow signals.

A key aspect in the calculation of fSampEn is the choice of the window size and the $r$ parameter. Since the $r$ parameter depends on the LS_SD, we firstly analyzed the variability of LS_SD of all the LS signals recorded. High intra-subject variability was found due to different flow levels generated during the inspiratory threshold loading protocol. Some intersubject variability was also found between healthy subjects and COPD patients, probably due to the difficulty of patients to generate flows as high as those of healthy subjects during threshold loading. Despite the variability of LS_SD values, a unique global LS_SD value was required in order to compare fSampEn time-series of LS signals in different subjects and at different flow levels. Regarding window length, in general, the longer the window, the smoother the fSampEn time-series. Long windows are therefore desirable to minimize the influence of artifacts in LS signals. Moreover, since fSampEn time-series were compared with flow signals, which are lowfrequency signals and vary slowly over time, longer windows performed better than shorter windows.

In summary, we performed a thorough evaluation of the most relevant parameters of fSampEn ( $r$ and window length), for a proper estimation of LSI in LS signals. The evaluation of fSampEn performed in a heterogeneous study dataset, allowed us to propose general values for fSampEn parameters that permit optimal calculation and comparison of fSampEn timeseries of LS signals in different subjects, thus contributing to improving the assessment of LSI in health and disease.

\section{REFERENCES}

A. R. A. Sovijärvi et al., "Characteristics of breath sounds and adventitious respiratory sounds," Eur. Respir. Rev., vol. 10, no.
77, pp. 591-596, 2000.

[2] N. Gavriely, M. Nissan, A. H. E. Rubin, and D. W. Cugell, "Spectral characteristics of chest-wall breath sounds in normal subjects," Thorax, vol. 50, pp. 1292-1300, 1995.

[3] A. Bohadana, G. Izbicki, and S. S. Kraman, "Fundamentals of lung auscultation," N. Engl. J. Med., vol. 370, no. 8, pp. 744-51, 2014.

[4] J. A. Fiz, R. Jané, M. Lozano, R. Gómez, and J. Ruiz, "Detecting unilateral phrenic paralysis by acoustic respiratory analysis," PLoS One, vol. 9, no. 4, p. e93595, Jan. 2014.

[5] L. Sarlabous, A. Torres, J. A. Fiz, and R. Jané, "Evidence towards improved estimation of respiratory muscle effort from diaphragm mechanomyographic signals with cardiac vibration interference using sample entropy with fixed tolerance values," PLoS One, vol. 9, no. 2, p. e88902, 2014.

[6] L. Estrada, A. Torres, L. Sarlabous, and R. Jané, "Improvement in neural respiratory drive estimation from diaphragm electromyographic signals using fixed sample entropy," IEEE $J$. Biomed. Heal. Informatics, vol. 20, no. 2, pp. 476-485, 2016.

[7] M. Lozano-García et al., "Surface mechanomyography and electromyography provide non-invasive indices of inspiratory muscle force and activation in healthy subjects," Sci. Rep., vol. 8, no. 1, p. 16921, Dec. 2018.

[8] J. S. Richman and J. R. Moorman, "Physiological time-series analysis using approximate entropy and sample entropy," Am. J. Physiol. Hear. Circ. Physiol., vol. 278, no. 6, pp. 2039-2049, 2000.

[9] L. Estrada, A. Torres, L. Sarlabous, and R. Jané, "Influence of parameter selection in fixed sample entropy of surface diaphragm electromyography for estimating respiratory activity," Entropy, vol. 19, no. 9, p. 460, Sep. 2017.

[10] M. Lozano-García, L. Estrada, and R. Jané, "Performance evaluation of fixed sample entropy in myographic signals for inspiratory muscle activity estimation," Entropy, vol. 21, no. 2, p. 183, Feb. 2019.

[11] American Thoracic Society/European Respiratory Society, "ATS/ERS Statement on respiratory muscle testing," Am. J. Respir. Crit. Care Med., vol. 166, no. 4, pp. 518-624, Aug. 2002.

[12] S. Huq and Z. Moussavi, "Acoustic breath-phase detection using tracheal breath sounds.," Med. Biol. Eng. Comput., vol. 50, no. 3, pp. 297-308, Mar. 2012.

[13] B. Reyes, N. Reljin, and K. Chon, "Tracheal sounds acquisition using smartphones," Sensors, vol. 14, no. 8, pp. 13830-13850, Jul. 2014.

[14] L. Yu et al., "Using the entropy of tracheal sounds to detect apnea during sedation in healthy nonobese volunteers," Anesthesiology, vol. 118, no. 6, pp. 1341-1349, Jun. 2013.

[15] A. Yadollahi and Z. M. K. Moussavi, "A robust method for estimating respiratory flow using tracheal sounds entropy.," IEEE Trans. Biomed. Eng., vol. 53, no. 4, pp. 662-8, Apr. 2006.

[16] Y. Castillo-Escario, I. Ferrer-Lluis, J. M. Montserrat, and R. Jané, "Entropy analysis of acoustic signals recorded with a smartphone for detecting apneas and hypopneas: a comparison with a commercial system for home sleep apnea diagnosis," IEEE Access, vol. 7, pp. 128224-128241, 2019. 